\title{
Seasonality modeling of the distribution of Aedes albopictus in China based on climatic and environmental suitability
}

\author{
Xueli Zheng ${ }^{1 *}$ D, Daibin Zhong ${ }^{2}$, Yulan $\mathrm{He}^{1}$ and Guofa Zhou ${ }^{2}$
}

\begin{abstract}
Background: Aedes albopictus is a highly invasive mosquito species and a major vector of numerous viral pathogens. Many recent dengue fever outbreaks in China have been caused solely by the vector. Mapping of the potential distribution ranges of Ae. albopictus is crucial for epidemic preparedness and the monitoring of vector populations for disease control. Climate is a key factor influencing the distribution of the species. Despite field studies indicating seasonal population variations, very little modeling work has been done to analyze how environmental conditions influence the seasonality of Ae. albopictus. The aim of the present study was to develop a model based on available observations, climatic and environmental data, and machine learning methods for the prediction of the potential seasonal ranges of Ae. albopictus in China.
\end{abstract}

Methods: We collected comprehensive up-to-date surveillance data in China, particularly records from the northern distribution margin of Ae. albopictus. All records were assigned long-term (1970-2000) climatic data averages based on the WorldClim 2.0 data set. Machine learning regression tree models were developed using a 10-fold crossvalidation method to predict the potential seasonal (or monthly) distribution ranges of Ae. albopictus in China at high resolution based on environmental conditions. The models were assessed based on sensitivity, specificity, and accuracy, using area under curve (AUC). WorldClim 2.0 and climatic and environmental data were used to produce environmental conduciveness (probability) prediction surfaces. Predicted probabilities were generated based on the averages of the 10 models.

Results: During 1998-2017, Ae. albopictus was observed at 200 out of the 242 localities surveyed. In addition, at least 15 new Ae. albopictus occurrence sites lay outside the potential ranges that have been predicted using models previously. The average accuracy was $98.4 \%$ (97.1-99.5\%), and the average AUC was $99.1 \%$ (95.6-99.9\%). The predicted Ae. albopictus distribution in winter (December-February) was limited to a small subtropical-tropical area of China, and Ae. albopictus was predicted to occur in northern China only during the short summer season (usually June-September). The predicted distribution areas in summer could reach northeastern China bordering Russia and the eastern part of the Qinghai-Tibet Plateau in southwestern China. Ae. albopictus could remain active in expansive areas from central to southern China in October and November.

Conclusions: Climate and environmental conditions are key factors influencing the seasonal distribution of Ae. albopictus in China. The areas predicted to potentially host Ae. albopictus seasonally in the present study could reach northeastern China and the eastern slope of the Qinghai-Tibet Plateau. Our results present new evidence and suggest the expansion of systematic vector population monitoring activities and regular re-assessment of epidemic risk potential.

Keywords: Aedes albopictus, Distribution, Northern margin, China, Climate, Environment

\footnotetext{
* Correspondence: zhengxueli2001@126.com

'Department of Pathogen Biology, School of Public Health, Southern Medical

University, 1838 Guangzhou North Avenue, Guangzhou 510515, China

Full list of author information is available at the end of the article
}

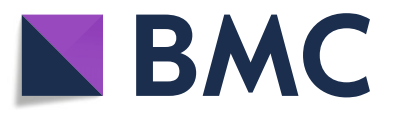

(c) The Author(s). 2019 Open Access This article is distributed under the terms of the Creative Commons Attribution 4.0 International License (http://creativecommons.org/licenses/by/4.0/), which permits unrestricted use, distribution, and reproduction in any medium, provided you give appropriate credit to the original author(s) and the source, provide a link to the Creative Commons license, and indicate if changes were made. The Creative Commons Public Domain Dedication waiver (http://creativecommons.org/publicdomain/zero/1.0/) applies to the data made available in this article, unless otherwise stated. 


\section{Multilingual abstracts}

Please see Additional file 1 for translations of the abstract into the five official working languages of the United Nations.

\section{Background}

Aedes albopictus (Stegomyia albopicta) Skuse, a mosquito native to the tropical and subtropical areas of south and east Asia, is an epidemiologically important vector of numerous viral pathogens, including yellow, dengue, chikungunya, and potentially Zika fever viruses [1-4]. An aggressive invasive species, Ae. albopictus has invaded and adapted to diverse environments in numerous countries across all continents except Antarctica [5]. The frequent outbreaks of dengue and chikungunya fevers in southern and southeastern China over the past few decades, and in central and eastern China in the past few years have raised major public health concerns [6-8]. In numerous cases, Ae. albopictus has been the sole vector responsible for dengue fever outbreaks in China [8, 9]. At present, vaccines are available for some Aedes mosquito transmitted diseases such as yellow fever and dengue fever. However, Sanofi Pasteur, the dengue fever vaccine manufacturer, announced in 2017 that people who receive the vaccine and have not been infected previously with a dengue virus may be at risk of developing severe dengue fever if they contract dengue after being vaccinated (https:// www.cdc.gov/dengue/prevention/dengue-vaccine.html).

In addition, no therapeutic treatments are available for most viruses transmitted by Ae. albopictus, which makes vector control a key strategy for controlling the transmission of such diseases [5]. Therefore, understanding the biology, distribution, and factors influencing the expansion of its range could facilitate the formulation of effective vector control strategies.

Local environmental conditions are key factors influencing the distribution, survivorship, and development of both larval and adult Ae. albopictus mosquitoes [10, 11]. The high capacity of Ae. albopictus to adapt to diverse environments and climates makes it a potential invasive species in numerous localities globally [1-5, 12-15]. The dynamic expansion in range raises questions about how environmental factors influence the distribution of $A e$. albopictus. For example, can Ae. albopictus extend its distribution range to temperate regions such as northern China? If the answer is 'Yes', since winters are very cold in northern China, can Ae. albopictus adults survive the winters or only emerge in summer in northern China?

Numerous studies have mapped regional or global distribution ranges of Ae. albopictus based on the biological or physiological characteristics of the species. Majority of the studies have examined how climatic conditions limit the distribution of the species, often focusing on temperature exclusively. Some studies have used laboratory-based results to predict how climatic factors, i.e., temperature and precipitation, affect the northern limits of Ae. albopictus in different regions [16-19] and potential ranges of Ae. aegypti and Ae. albopictus under present-day and future climate conditions [20]. Such studies present the most recent trends in research on Aedes risks. It is essential to examine how local environments influence Ae. albopictus ranges, particularly their seasonality in China, based on a more comprehensive review of field observational data since it could provide insights into the underlying factors $[9,13]$. Prediction of seasonality is particularly critical because could facilitate better allocation of resources and guarantee cost-effectiveness in disease prevention and control.

In China, which has a vast territory, most regions are located in the temperate zone while some southern regions are located in the subtropical and tropical zones [21]. The northern region is close to the frigid zone. The Qinghai-Tibetan Plateau in southwest China is an area experiencing low temperatures throughout the year. There are also arid and semi-arid climates in the northwestern desert regions [21]. Notably, previous climatic suitability studies suggested that Ae. albopictus would be found only in the tropical, sub-tropical and warm temperate areas of China [20-22]. In a systematic surveillance that covered 19 provinces from 2006 to 2013, Ae. albopictus individuals were trapped in 16 out of the 19 provinces [23], which was consistent with previous reports [20, 22]. However, a more comprehensive review with updated information is required to evaluate the current distribution limits of Ae. albopictus in China.

Field studies from different areas have delineated high seasonality in Ae. albopictus population dynamics and distribution. For example, in Croatia, Ae. albopictus oviposition activity began in April and ended in November, with high seasonality and spatial heterogeneity [24]. In the northern temperate areas of Japan, adult Ae. albopictus were observed from May through October [25-28]. Similarly, in southern China, Ae. albopictus were active all year round [29]; however, in central China, adult Ae. albopictus were observed only from May to October [30]. Despite such findings in the field, modeling studies have rarely analyzed the seasonal influence of environment on Ae. albopictus populations and their distribution [18, 22, 31, 32].

In the present study, our aim was to model the seasonal distribution of Ae. albopictus in China based on comprehensive data from published literature. Our models used environmental data to predict distribution and seasonality, particularly in the earliest and latest months of occurrence of the species, at high spatial resolution. The present study focuses solely on the distribution of adult Ae. albopictus.

\section{Methods}

Comprehensive Ae. albopictus field surveillance records and GPS location data from China for the 1998-2017 
period were collected (Additional file 2: Table S1 and Additional file 3: Table S2). Since Ae. albopictus has been observed frequently in southern China [7-10], to minimize redundancy during modeling, repeated sampling activities in similar locations (e.g., the same city) in southern China (e.g., Guangdong, Zhejiang, and other provinces) were not included [7-10]. Information extracted from the surveillance records included prevalence and months of occurrence of Ae. albopictus. Incomplete occurrence period records were filled in using $\mathrm{k}$-medoids clustering based on climate similarity (see Data analysis below). If a specific location was included in the published record, the GPS readings of that record were extracted. If only the name of the sampling location was included (usually the name of the city or county where the sample was collected), a GPS location was arbitrarily selected within the city or the major town (usually the capital) of the county. The GPS readings were used to extract the climatic and environmental data described in the following section.

\section{Climatic and environmental data for model development}

We used WorldClim 2.0 as major reference climatic data [33]. High resolution (30 arcsec or approximately $1-\mathrm{km}^{2}$ spatial resolution) WorldClim 2.0 data were generated based on averages during 1971-2000 [33]. Climatic data variables included monthly mean, minimum, and maximum temperatures, and monthly total precipitations. Climatic data that assigned to each sampling point were from the nearest grid points in WorldClim 2.0. Climatic-environmental zones were established based on previous literature [21]. The environmental regions were divided into four categories, including humid, sub-humid, semiarid, and arid regions. Climatic zone comprised nine categories, including south subtropical, mid-subtropical, north subtropical, warm temperate, mild temperate, cool temperate, plateau subtropical, plateau temperate, and plateau sub frigid zones. Each sampling location was assigned its corresponding climatic and environmental categories.

\section{Climatic data for spatial prediction}

To map the spatial distribution of Ae. albopictus based on climatic-environmental factors in China, we used existing globally gridded climate surfaces WorldClim 2.0 data in the present study [33]. The WorldClim dataset has used extensively for the modeling of the impact of global climate change on the distribution of Ae. albopictus, particularly its range expansion, as well as the mapping of dengue and chikungunya fever risks in the US, Europe, and globally $[13,19,31,34]$. WorldClim2.0 data includes globally gridded climate surfaces ( 30 arcsec or approximately $1-\mathrm{km}^{2}$ spatial resolution) of monthly average temperature (minimum, mean, and maximum) and monthly rainfall for 1971-2000.

\section{Data analysis}

Data analysis included three steps. Step 1: assigning climatic and environmental data to each surveillance record based on the GPS location of the record, regardless of the status of occurrence of Ae. albopictus. Step 2: sorting sampling points into groups based on similarity in environmental conditions and the known Ae. albopictus occurrence months, then assigning occurrence months to missing records due to lack of longitudinal observations, which was carried out using k-medoids clustering [35]. Step 3: predicting the conduciveness of environmental conditions for Ae. albopictus populations using a machine-learning regression model. The model was evaluated by examining Ae. albopictus occurrence against model-predicted probability of occurrence at each sampling site in each month. The cutoff predicted probability for determining 'presence' or 'absence' was based on accuracy [36], which balances specificity and sensitivity. The accuracy is calculated as the sum of correctly predicted positives and correctly predicted negatives over a total number of samples. The eventual model sensitivity and specificity were calculated based on a cutoff predicted probability that gives the optimal accuracy, i.e., if the predicted risk rate was greater than cutoff, the prediction was considered 'presence'; otherwise it was considered 'absence'. Sensitivity was estimated based on the proportion of sites with Ae. albopictus that were predicted as 'presence' and specificity was estimated based on the proportion of sites without Ae. albopictus that were predicted as 'absence'. In addition to sensitivity, specificity, and accuracy, we also calculated area under curve (AUC) based on receiver operating characteristic curve or ROC curve. AUC provides an aggregate measure of performance across all possible classification thresholds. Subsequently, the predicted monthly Ae. albopictus occurrence maps were generated.

The following are briefs of the k-medoids clustering. kmedoids is a classical partitioning clustering technique that clusters a dataset of objects into $\mathrm{k}$ groups known a priori [35]. The k-medoid clustering applied most extensively is the Partitioning Around Medoids (PAM) algorithm [35], which was selected for the present study. The method initially selects $\mathrm{k}$ data points as the medoids, and the points represent different clusters. Each data point is associated with the closest medoid through a stepwise method to minimize the cost of the configuration, i.e., sum of distances of points to their medoid. Briefly, for each medoid and non-medoid data point, swap medoid with non-medoid, and re-compute the cost. If the total cost of the configuration decreases, then continue, otherwise undo the swap. The process continues until all data points are assigned to clusters. In the study, we knew that Ae. albopictus occurred all-year-around in tropical China, in regions such as Hainan Island; however, one may 
observed adult Ae. albopictus only in summer from June to September in northern China, in regions such as in Liaoning province (Additional file 2: Table S1, Additional file 3: Table S2 and Additional file 4: Figure $\mathrm{S} 1)$. The initial $\mathrm{k}$ value was set as 12 , and values including $11,10,9$, and 8 were also examined. We used data points with known Ae. albopictus occurrence months as training data then used clustering results to assign occurrence months to each record in cases where they were missing due to a lack of longitudinal observations.

The machine learning classification and regression tree (CART) analysis was used to predict the monthly/seasonal distribution of Ae. albopictus. The binary values, 0 and 1, representing 'presence' and 'absence', respectively were assigned to each month for all sampling points based on field observation or the results of k-medoids clustering. The binary variables were then used for CART modeling as the dependent variables, while environmental variables were the independent variables. We have adapted the commonly used 10-fold cross-validation rule in the present study $[37,38]$. Consequently, 10 prediction models were produced for each month. The training sets were used to grow the tree and the testing sets were used for cross-validation to prune the tree. We set a minimum data points of five for all terminate nodes. Since Ae. albopictus was only present in a small area in southern China in winter (December-February) and absent in a small area in northern China in summer (July-September), the stratification (i.e., rearrangement of the data) 10 -fold cross-validation method was used to ensure that each fold was an appropriate representative of the whole to minimize sampling bias induced by 10 -fold random selection [37, 38].

Once the models were established, climatic-environmental suitability maps were generated for each month based on climatic surface, climatic zone, and regional environmental data. Suitability was predicted as the average predicted suitability probability of the 10 models developed during the 10fold cross-validation modeling process, and spatial resolution was 30 arcsec or approximately $1 \mathrm{~km}^{2}$ spatial resolution.

All data analyses were conducted and maps generated using the open-source programming language R v3.3.2 ( $R$ Foundation for Statistical Computing, Vienna, Austria). For $k$-medoids clustering, we used the pam method of the cluster package; for raster image reading and risk mapping, we used the raster and crop methods within the rasterImage and $s p$ packages; and for regression tree modeling, we used the ctree and rpart methods within the rpart, party, and caret packages.

\section{Results}

\section{Updated Ae. albopictus distribution map in China}

Two hundred and forty-two sampling locations were included in the present study (Fig. 1). Ae. albopictus was recorded in 200 sites and no Ae. albopictus were observed in the rest of the 42 sites. Only four provinces had ongoing systematic surveillance programs, including two southern provinces, Zhejiang and Hainan Island, representing the subtropical-tropical areas; one southwestern province, Guizhou, which is a high-elevation mountainous area; and Shaanxi province, representing northern China, and potentially the northern distribution margin of Ae. albopictus in China (Fig. 1, Additional file 4: Figure S1) [39-42]. Data records included 31 sampling sites (county or city) in Shaanxi province, in which Ae. albopictus occurred in 20 sites (Fig. 1, Additional file 4: Figure S1). Gansu province, in the semiarid and arid area of northwestern China, had 22 sampling sites, of which 14 had Ae. albopictus (Fig. 1). No Ae. albopictus were observed in the Ningxia Hui Autonomous Region, the Inner Mongolia Autonomous Region, or Qinghai province, which are all located in the semiarid, arid or Qinghai-Tibetan Plateau temperate areas (Fig. 1). No sampling has been conducted previously in the Tibet Autonomous Region, and very few collections have been carried out in Qinghai province, and the Xinjiang Uygur Autonomous Region; the three provinces represent some of the provinces with the largest land areas and the harshest climatic and environmental conditions in western China (Additional file 4: Figure S1). Most of the Ae. albopictus positive sites in the present study are in areas where ,the species have been found before, and the areas are considered to have suitable climatic conditions (Fig. 1, Additional file 4: Figure S1) [20, 22, 23, 32]. Notably, however, some more recent reports of Ae. albopictus are far outside the conventional suitable climate ranges including a site in Heilongiiang province in northeastern China, which borders the Democratic People's Republic of Korea, a site in the far west of Gansu province, in the desert city of Jiayuguan, and a site in northern Xinjiang, which borders Russia, Mongolia and Kazakhstan (Fig. 1). There are also several Ae. albopictus positive sampling sites in northern Liaoning province, in which the vector has never been reported previously, in addition to two sites on the east slope of the Qinghai-Tibet Plateau in western Sichuan province (Fig. 1, Additional file 4: Figure S1).

\section{Seasonal risk modeling}

Climatic and environmental similarity analysis results suggested that the observational data points could be clustered into nine clusters because all the Ae. albopictus occurrence sites corresponded to the June-September period; therefore, regression trees were developed for 9 months or clusters of months, with June-September considered one cluster. Figure 2 illustrates the sensitivity, specificity, and accuracy of the models, in addition to the AUC (Fig. 2, Additional file 5). Using a cutoff yield the optimal accuracy for each month or cluster of months. The average model accuracy was $98.4 \%$ (range: 97.1-99.9\%) and the average AUC was 99.1\% (range: 


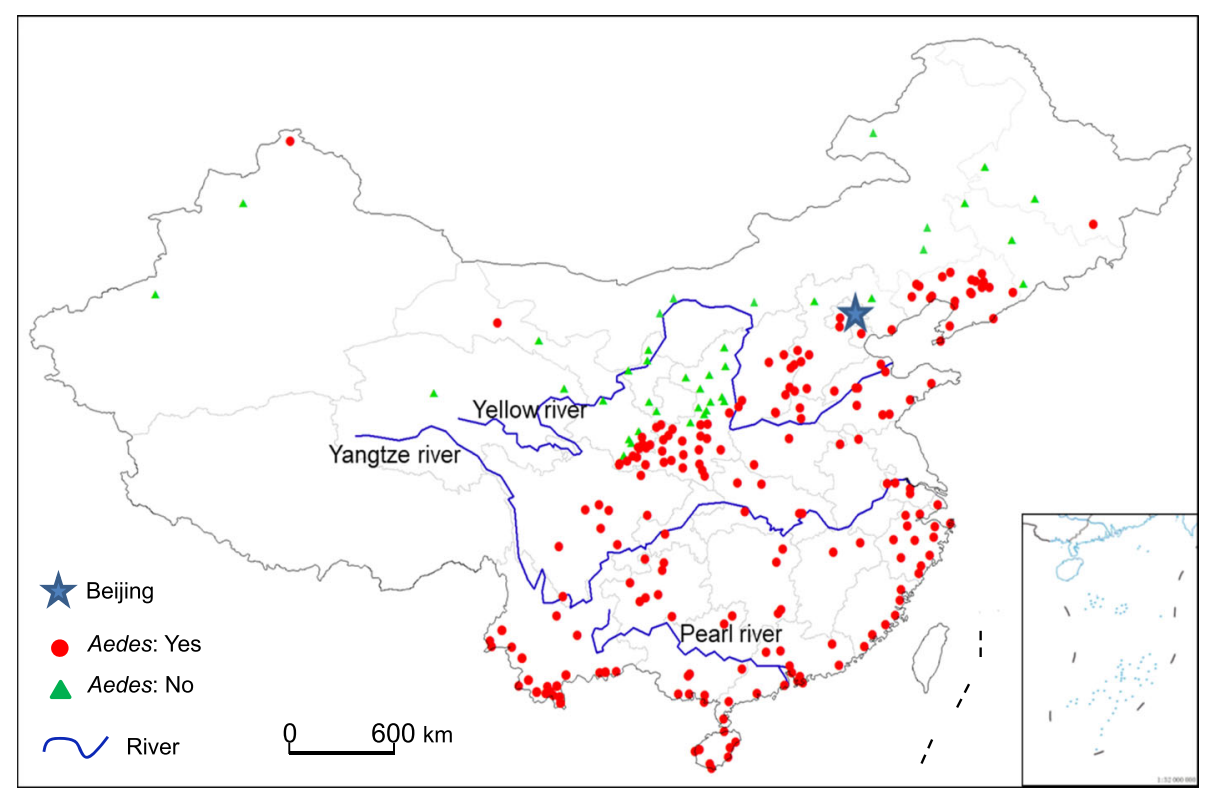

Fig. 1 Map of Aedes albopictus surveillance sites in China. Bottom right box: South China Sea islands

95.7-99.9\%). The sensitivity rate was $97.2 \%$ on average (range: 94.4-98.2\%), with the lowest value in January and the highest in April. The specificity rate was 99.1\% on average (range: 97.1-100\%).

\section{Seasonal risk mapping}

The model unequivocally predicts that in the winter months (December-February) Ae albopictus will be observed only in southern China, as depicted in the risk maps (Fig. 3). The major expansion of the range of Ae. albopictus adults begins in April, when the predicted occurrence covers most of the areas south of the Yellow River (Fig. 3). By May, it covered all of China, including a few places in northwestern China, although risk probabilities are low in the northeast and southwest regions (Fig. 3). In JuneSeptember, the major dengue fever transmission season in China, the model predicts the greatest range of climate and environmental suitability. The most notable areas are included northeastern China, and the eastern slope of the Qinghai-Tibet Plateau in southwestern China (Fig. 3), which have been predicted in previous studies to have unsuitable climates. Predicted ranges decreased considerably by October compared to June-September, when the risk map is similar to the map of April. In November, Ae. albopictus adults remained active across most regions south of the Yangtze River and in a small portion of the areas north of the Yangtze River (Fig. 3).

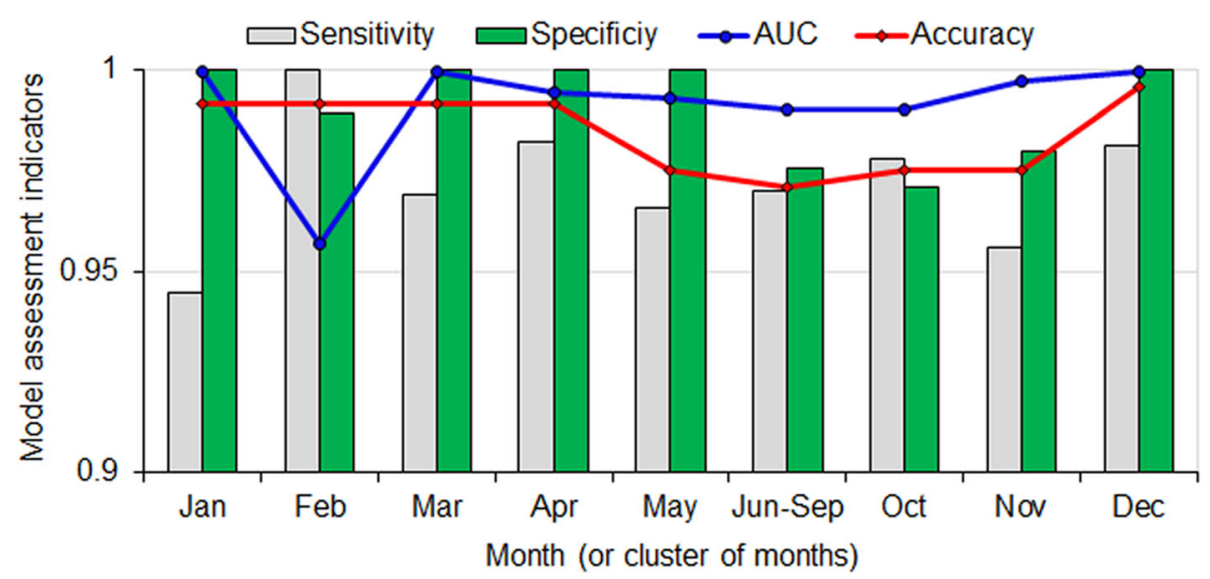

Fig. 2 Model sensitivity, specificity, accuracy, and area under curve based on months 


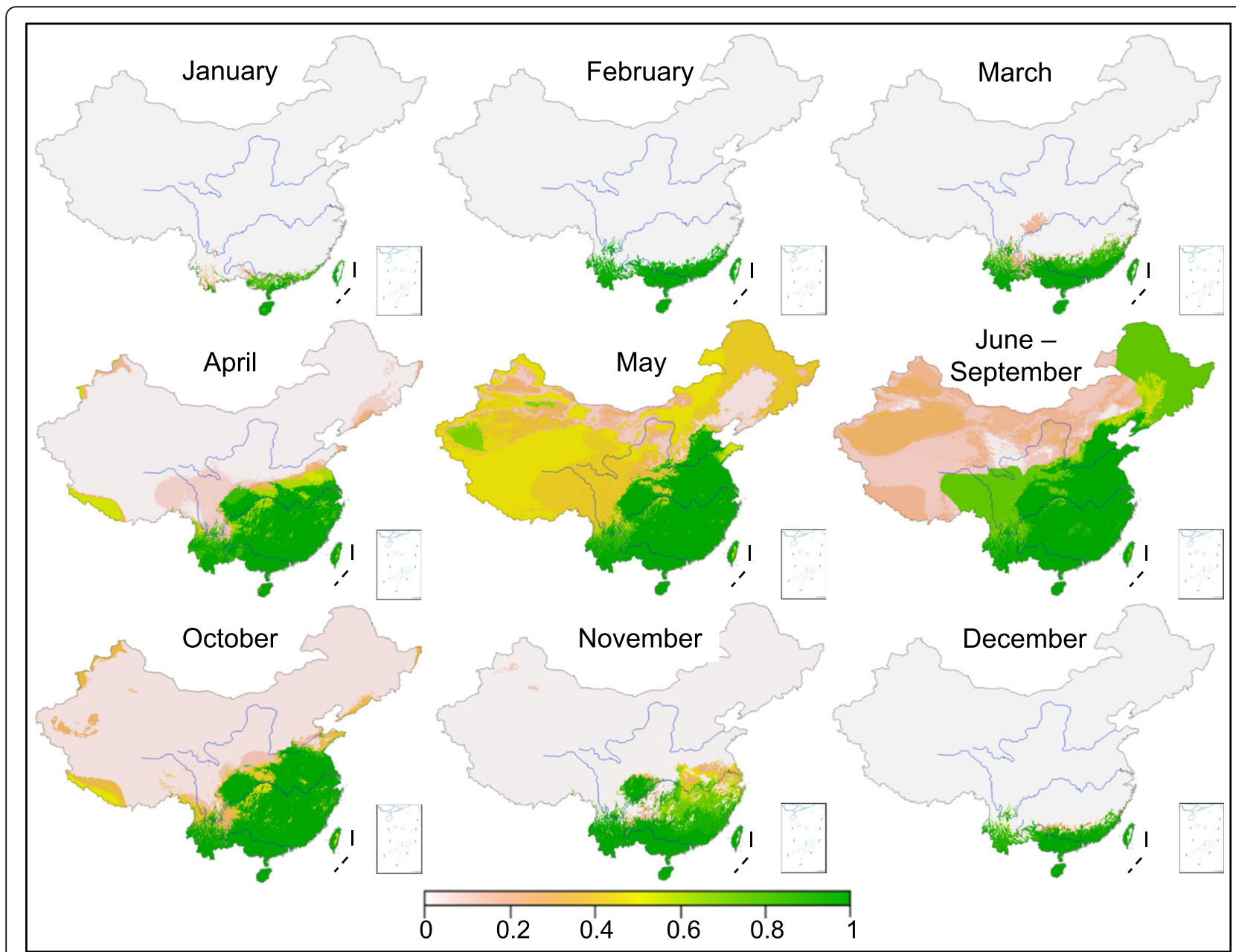

Fig. 3 Maps of Aedes albopictus risk probabilities based on months or clusters of months. Blue curves are the major rivers in China from north to south: Yellow (Huanghe), Yangtze (Changjiang), and Pearl (Zhujiang) rivers. There was no prediction for the South China Sea islands; therefore, South China Sea islands are not showing displayed in the graph (see Fig. 1, bottom right box)

\section{Discussion}

Ae. albopictus has extended its range globally due to its capacity to adapt to the environment and climate change [1-5]. The exploration of the environmental factors influencing its distribution range in China could facilitate the formulation of effective monitoring and risk assessment programs, since new dengue fever outbreaks are a threat to public health. Indeed, recent dengue fever epidemics have occurred in different regions ranging from Guangzhou, in southern China, in 2014, to Yuzhou in Henan province, which is on the south bank of the Yellow River in central China, $1400 \mathrm{~km}$ straight north of Guangzhou, in 2013 [43]. Almost all the recent epidemics were caused solely by Ae. albopictus $[6-9,43]$. The 2013 dengue fever epidemic in Yuzhou, initiated by an imported index case, pointed to the suitability of the local environment-climate in central China. The danger lies in the fact that imported cases have been reported all over China [44]; therefore, there is a need to update the vector distribution maps and reassess environmental suitability based on the updated distribution data. Although our analysis introduced new sampling sites in China, we anticipated Ae. albopictus distributional potential similar to that reported in the previous studies $[20,22]$. The five most notable sites included two in northwestern China (Jiayuguan City in Gansu and Beitun City in Xinjiang) and one in northeastern China (Ning'an County in Heilongjiang), and two sites in Jiulong and Lixian counties in western Sichuan province, which are located at high altitude (close to $3000 \mathrm{~m}$ above sea level) on the east slope of the Qinghai-Tibetan Plateau (Fig. 1). Using environmental data from the new sites, the present study generated a new risk map with risk ranges extended considerably in summer, particularly in northeastern China in areas bordering Russia and on the eastern slope of the Qinghai-Tibetan Plateau in southwestern China. The predicted regions with suitable climates from May to September extended beyond 
the areas predicted substantially even in other recent studies [22, 32]. Whether or not the expansion in the Ae. albopictus population range is linked to global climate change or the biological adaptation of vectors requires further study.

Based on the updated distribution data, the present study predicted that Ae. albopictus could occur seasonally in semiarid or even arid areas, as long as temperature and precipitation conditions are favorable, which suggests a need to consider seasonal climate suitability as opposed to focusing on specific annual periods. In addition to temperature, water availability influences mosquito distribution [45]. In most temperate areas of China, precipitation is highly seasonal and temperatures are usually high in summer [21, 22]. The short summers, relatively high temperature, and seasonal rainfall in northern China could facilitate the establishment of Ae. albopictus populations in the region, which has occurred in Japan in regions at similar latitudes and in the northeastern United States $[46,47]$. With regard to the occurrence of Ae. albopictus in semiarid and arid areas, the key examples are Pakistan and Saudi Arabia, where dengue fever outbreaks have been reported previously $[46,47]$.

Other Ae. albopictus risk areas of interest are the southwestern international border areas and the eastern slope of the Qinghai-Tibetan Plateau. Could the environmental conditions in such regions facilitate Ae. albopictus population establishment? In the present study, five Ae. albopictus occurrence sites had elevations > $2000 \mathrm{~m}$ above sea level, with the highest at approximately $2900 \mathrm{~m}$. Dengue fever epidemics and Ae. albopictus have been reported in northern Pakistan, not far from the China-Pakistan border [46, 47]. In addition, dengue fever epidemics and both Ae. albopictus and Ae. aegypti have been reported in Nepal [48-50], which lies on the south slope of the Himalaya mountain range, also bordering southwestern China. Such studies indicate that vectors of tropical infectious diseases could adapt to the environmental conditions in the low altitude section of the Qinghai-Tibetan Plateau and could even transmit the diseases there. Again, the influence of global climate change on such dynamics is a subject for further study.

The present study has some major limitations. The model applied climatic data from 1971 to 2000, which may have limited its capacity to predict the recent expansion of Ae. albopictus ranges, although WorldClim data has been widely used for the prediction of the impact of global change on the expansion of Ae. albopictus ranges $[13,19,31,34]$. Since climate change has intensified over the past two decades (http://www.ncdc.noaa. gov/cag/), incorporating the most recent data could yield an even more substantial Ae. albopictus range expansion or late-year (late fall or early winter) activity predictions $[18,31]$. For example, the present study predicted that
Ae. albopictus adult activity would be restricted to the south of the Yellow River by October and to the south of the Yangtze River by November.

A previous study conducted in Shandong province, along the banks of the Yellow River, trapped Ae. albopictus outdoors in November [51], which our model predicted as 'absence' with zero probability. This could be due to 1) old climatic data used in the modeling activity; 2) annual climate variability; or 3) real temperature increase or climate change, which warrant further investigation. Another limitation of the present study is the lack of human distribution data [52-54]. While environmental factors influence vector population growth, human population distribution influences the transmission of disease [54, 55]. In the arid areas of northern and northwestern China and in numerous parts of the Qinghai-Tibetan Plateau, the human population distribution is sparse; therefore, potential dengue fever risks are low, particularly in the frigid area on top of the plateau. However, how climate and environmental factors interact with human distribution in landscapes and biological competitors, and their combined influence on $A e$. albopictus population establishment and distribution require further investigation. Since the sampling sites in the present study were point data, further analyses, such as spatial extrapolation studies, are required, so that expansion ranges and increased risks in populations can be estimated in combination with population density maps. A further prospect would be analyzing local climate trends in the past and linking them with observed dengue fever outbreaks, which would be more appealing from a public health perspective than simply focusing on vector distribution ranges [1-5]. Furthermore, when mapping the distribution and seasonality of Ae. albopictus and dengue fever outbreaks in China, it could be more appropriate to use only meteorological data from stations in China and international stations near the borders. This warrants further explorations and it is the objective of our ongoing project.

Finally, systematic sampling, i.e., good spatial coverage and sufficient longitudinal follow-ups, is key for the determination of the distribution ranges conduciveness of climatic conditions for Ae. albopictus. Currently, systematic survey data on Ae. albopictus are only available for four provinces in China [39-42]. Although China's Center for Disease Control and Prevention has implemented a cross-country surveillance system and conducted longitudinal surveys, the spatial coverage is too coarse for a comprehensive nationwide evaluation of $A$. albopictus distribution [6, 23]. June-September is an important season for mosquito population dynamics monitoring, based on the results of this study. We emphasize that both spatial coverage and sampling seasons are essential for determining the northern geographic margins of distribution of the vector. 


\section{Conclusions}

Both climatic and environmental factors both influence Ae. albopictus distribution in China. The current predicted seasonal distribution of Ae. albopictus in China may extend well beyond the ranges predicted previously, particularly the northern limit, due to either global climatic change or the adaptation of biological vectors. Considering the sustained movement of dengue fever epidemics northward in central and eastern China, the timely monitoring of Ae. albopictus populations and their expansion, the assessment, and frequent reassessment of epidemic risks in such areas, and the assessment of risks based on recent dengue fever outbreaks in China, particularly in central and northern China, are crucial for epidemic preparedness and disease control, particularly in the wake of global climate change.

\section{Supplementary information}

Supplementary information accompanies this paper at https://doi.org/10. 1186/s40249-019-0612-y.

Additional file 1. Multilingual abstracts in the five official working languages of the United Nations.

Additional file 2: Table S1. Reference list for Aedes albopictus surveillance records.

Additional file 3: Table S2. List of Aedes albopictus surveillance locations used in the present study.

Additional file 4: Figure S1. Map of China with provinces and their boundaries. Bottom right box: South China Sea islands.

Additional file 5: Figure S2. Graphs of receiver operating characteristic curves (ROCs) for each month or cluster of months.

\section{Acknowledgements}

We thank Di-Zi Yang for her assistance with part of the data collections.

\section{Authors' contributions}

$X L Z$ and $G Z$ conceived and designed the study; $X L Z, G Z$, and $Y H$, participated in the collection of surveillance records and climatic data. GZ and YH conducted the data analysis. XLZ, DZ, and GZ drafted the manuscript. All authors were involved in the interpretation and discussion of the results and provided comments. All authors read and approved the final version of the manuscript.

\section{Funding}

This study was supported by grants from the National Natural Science Foundation of China (No: 31630011). Science and Technology Plan Project of Guangzhou city (No. 201804020084) and Natural Science Foundation of Guangdong province (No. 2017A030313625). The funders had no role in study design, data collection and analysis, decision to publish, or preparation of the manuscript.

\section{Availability of data and materials}

The data sets supporting the results are included within the article and Additional file 1.

\section{Ethics approval and consent to participate}

This study did not involve vertebrate animals or human subjects. No field study was conducted.

\section{Consent for publication}

No other personal data of any form were used in this study.

\section{Competing interests}

The authors declared that they have no competing interests.

\section{Author details}

'Department of Pathogen Biology, School of Public Health, Southern Medical University, 1838 Guangzhou North Avenue, Guangzhou 510515, China.

${ }^{2}$ Program in Public Health, University of California, Irvine, CA, USA.

Received: 15 July 2019 Accepted: 14 November 2019

Published online: 03 December 2019

\section{References}

1. Lambrechts L, Scott TW, Gubler DJ. Consequences of the expanding global distribution of Aedes albopictus for dengue virus transmission. PLoS Negl Trop Dis. 2010;4:e646.

2. Cao-Lormeau VM, Musso D. Emerging arboviruses in the Pacific. Lancet. 2014;384:1571-2.

3. Medlock JM, Hansford KM, Schaffner F, Versteirt V, Hendrickx G, Zeller $H_{\text {, }}$ et al. A review of the invasive mosquitoes in Europe: ecology, public health risks, and control options. Vector Borne Zoonotic Dis. 2012;12:435-47.

4. Weaver SC, Lecuit M. Chikungunya virus and the global spread of a mosquito-borne disease. N Engl J Med. 2015;372:1231-9.

5. Bonizzoni M, Gasperi G, Chen X, James A. The invasive mosquito species Aedes albopictus: current knowledge and future perspectives. Trends Parasitol. 2013:29:460-8.

6. Sun J, Lu L, Wu H, Yang J, Xu L, Sang S, et al. Epidemiological trends of dengue in mainland China, 2005-2015. Int J Infect Dis. 2017;57:86-91.

7. Wu D, Wu J, Zhang Q, Zhong H, Ke C, Deng X, et al. Chikungunya outbreak in Guangdong Province, China, 2010. Emerg Infect Dis. 2012;18:493-5.

8. Luo L, Jiang LY, Xiao XC, Di B, Jing QL, Wang SY, et al. The dengue preface to endemic in mainland China: the historical largest outbreak by Aedes albopictus in Guangzhou, 2014. Infect Dis Poverty. 2017;6:148.

9. Yang TC, Fu GM. Investigation on the distribution of dengue vector Aedes albopictus in Zhejiang province. Chinese J Hyg Insecticide Equip. 2006;12: 189-91.

10. Li Y, Kamara F, Zhou G, Puthiyakunnon S, Li C, Liu Y, et al. Urbanization increases Aedes albopictus larval habitats and accelerates mosquito development and survivorship. PLoS Negl Trop Dis. 2014;8:e3301.

11. Aida HN, Dieng H, Ahmad AH, Satho T, Nurita AT, Salmah MRC, et al. The biology and demographic parameters of Aedes albopictus in northern peninsular Malaysia. Asian Pac J Trop Biomed. 2011;1:472-7.

12. Hahn MB, Eisen L, McAllister J, Savage HM, Mutebi JP, Eisen RJ. Updated reported distribution of Aedes (Stegomyia) aegypti and Aedes (Stegomyia) albopictus (Diptera: Culicidae) in the United States, 1995-2016. J Med Entomol. 2017;54:1420-4

13. Cunze S, Kochmann J, Koch LK, Klimpel S. Aedes albopictus and its environmental limits in Europe. PLoS One. 2016;11:e0162116.

14. European Centre for Disease Prevention and Control. Mosquito maps: New information about areas with Aedes albopictus and Aedes aegypti in Europe. https://ecdc.europa.eu/en/news-events/mosquito-maps-new-informationabout-areas-aedes-albopictus-and-aedes-aegypti-europe.

15. Moore CG, Mitchell CJ. Aedes albopictus in the United States: ten-year presence and public health implications. Emerg Infect Dis. 1997;3:329-34.

16. Cunze S, Koch LK, Kochmann J, Klimpel S. Aedes albopictus and Aedes japonicus - two invasive mosquito species with different temperature niches in Europe. Parasit Vectors. 2016:9:573.

17. Kobayashi AM, Nihei N, Kurihara T. Analysis of northern distribution of Aedes albopictus (Diptera: Culicidae) in Japan by geographical information system. J Med Entomol. 2002;39:4-11.

18. Caminade C, Medlock JM, Ducheyne E, McIntyre KM, Leach S, Baylis M, et al. Suitability of European climate for the Asian tiger mosquito Aedes albopictus: recent trends and future scenarios. J R Soc Interface. 2012;9: 2708-17.

19. Campbell LP, Luther C, Moo-Llanes D, Ramsey JM, Danis-Lozano R, Peterson AT. Climate change influences on global vector distributions for dengue and chikungunya viruses. Phil Trans R Soc B. 2015;370:20140135.

20. Kamal M, Kenawy MA, Rady MH, Khaled AS, Samy AM. Mapping the global potential distributions of two arboviral vectors Aedes aegypti and Ae. albopictus under changing climate. PLoS ONE. 2018;13(12):e0210122.

21. Zheng J, Yin Y, Li B. A new scheme for climate regionalization in China. Acta Geograph Sin. 2010;65:3-13 (in Chinese).

22. Wu F, Liu Q, Lu L, Wang J, Song X, Ren D. Distribution of Aedes albopictus (Diptera: Culicidae) in northwestern China. Vector Borne Zoonotic Dis. 2011; 11:1181-6. 
23. Wu HX, Liu QY, Liu XB, Lu L, Guo YH, Yue YJ. Surveillance for Aedes albopictus in China, 2006-2013. Dis Surveill. 2015;30:310-5 (in Chinese).

24. Zitko T, Merdić E. Seasonal and spatial oviposition activity of Aedes albopictus (Diptera: Culicidae) in Adriatic Croatia. J Med Entomol. 2014;51: 760-8.

25. Sota T, Mogi M, Hayamizu E. Seasonal distribution and habitat selection by Aedes albopictus and Ae. riversi (Diptera: Culicidae) in northern Kyushu, Japan. Med Entomol. 1992;29:296-304.

26. Mogi M, Armbruster PA, Tuno N, Aranda C, Yong HS. The climate range expansion of Aedes albopictus (Diptera: Culicidae) in Asia inferred from the distribution of Albopictus subgroup species of Aedes (Stegomyia). J Med Entomol. 2017:54:1615-25.

27. Komagata O, Higa Y, Muto A, Hirabayashi K, Yoshida M, Sato T, et al. Predicting the start of the Aedes albopictus (Diptera: Culicidae) female adult biting season using the spring temperature in Japan. J Med Entomol. 2017; 54:1519-24.

28. Armstrong PM, Andreadis TG, Shepard JJ, Thomas MC. Northern range expansion of the Asian tiger mosquito (Aedes albopictus): analysis of mosquito data from Connecticut. USA PLoS Negl Trop Dis. 2017;11: e0005623.

29. Lin HS, Zhang W, Wang SW, Chen HS. Analysis and monitoring of dengue fever vector in Leizhou city in 2006. Chin J Vector Bio Control. 2008;19:464-6 (in Chinese).

30. Kong F, Wang H, Song H, Zheng J, Kong Y, Kong F. Survey of Aedes and its control at Confucious Forest in Qufu. Chin J Vector Biol Control. 2001;12 195-6 (in Chinese).

31. Tjaden NB, Suk JE, Fischer D, Thomas SM, Beierkuhnlein C, Semenza JC. Modelling the effects of global climate change on Chikungunya transmission in the 21st century. Sci Report. 2017;7:3813.

32. Kraemer MU, Sinka ME, Duda KA, Mylne AQ, Shearer FM, Barker CM, et al. The global distribution of the arbovirus vectors Aedes aegypti and $A e$ albopictus. Elife. 2015:4:e08347.

33. Fick SE, Hijmans RJ. WorldClim 2: new 1-km spatial resolution climate surfaces for global land areas. Int J Climatol. 2017;37:4302-15.

34. Escobar LE, Qiao H, Peterson AT. Forecasting Chikungunya spread in the Americas via data-driven empirical approaches. Parasit Vectors. 2016:9:112.

35. Kaufman L, Rousseeuw PJ. Finding groups in data: an introduction to cluster analysis. New York: Wiley; 1990.

36. Liu C, Berry PM, Dawson TP, Pearson RG. Selecting thresholds of occurrence in the prediction of species distributions. Ecography. 2005:28:385-93.

37. James G, Witten D, Hastie T, Tibshirani R. An Introduction to Statistical Learning: with Applications in R (Springer Texts in Statistics). 2017 Edition. Vienna: Springer; 2017

38. Kuhn M, Johnson K. Applied Predictive Modeling. 2018 Edition. Michigan: Springer; 2018.

39. Jin $Y M$, Wu $W X$, Sun $L Y$, Su XY, Jia PB, Li Z, et al. Survey on the distribution of Aedes mosquitoes transmitting dengue fever of Hainan Province in 2007. Chin Trop Med. 2008;8:2096-8 (in Chinese).

40. Shu LP, Zuo L, Zhao X, Chen AY, Wei LH. Susceptibility of 15 collections of Aedes albopictus from Guizhou to dengue virus oral infection. Chin J Exp Clin Virol. 2004;18:234-8 (in Chinese).

41. Fu GM, Yang TC, Ren ZY. Population surveillance of mosquitoes in Zhejiang province in 2006. Chin J Hyg Insect Equip. 2008;14:107-8 (in Chinese).

42. Han X, Zhang Y, Liu Z, Li L. Distribution of Aedes albopictus in Shaanxi province. Chin J Hyg Insect Equip. 2015:4:434-5 (in Chinese).

43. Huang XY, Ma HX, Wang HF, Du HF, Su J, Li XL, et al. Outbreak of dengue fever in Central China, 2013. Biomed Environ Sci. 2014;27:894-7.

44. Lai S, Huang Z, Zhou H, Anders KL, Perkins TA, Yin W, et al. The changing epidemiology of dengue in China, 1990-2014: a descriptive analysis of 25 years of nationwide surveillance data. BMC Med. 2015;13:100.

45. Alto BW, Juliano SA. Precipitation and temperature effects on populations of Aedes albopictus (Diptera: Culicidae): implications for range expansion. J Med Entomol. 2001:38:646-56.

46. Humphrey JM, Cleton NB, Reusken CBEM, Glesby MJ, Koopmans MPG, AbuRaddad LJ. Dengue in the Middle East and North Africa: A systematic review. PLoS Negl Trop Dis. 2016;10:e0005194 Rev.

47. Khan J, Khan I, Amin I. A comprehensive entomological, serological and molecular study of 2013 dengue outbreak of swat, Khyber Pakhtunkhwa Pakistan. PLoS One. 2016;11:e0147416.
48. Pandey BD, Nabeshima T, Pandey K, Rajendra SP, Shah Y, Adhikari BR, et al First isolation of dengue virus from the 2010 epidemic in Nepal. Trop Med Health. 2013;41:103-11.

49. Dhimal M, Gautam I, Joshi HD, O'Hara RB, Ahrens B, Kuch U. Risk factors for the presence of chikungunya and dengue vectors (Aedes aegypti and Aedes albopictus), their altitudinal distribution and climatic determinants of their abundance in Central Nepal. PLoS Negl Trop Dis. 2015;9:e0003545.

50. Dhimal M, Gautam I, Kreß A, Müller R, Kuch U. Spatio-temporal distribution of dengue and lymphatic filariasis vectors along an altitudinal transect in Central Nepal. PLoS Negl Trop Dis. 2014;8:e3035.

51. Jing X, Li Z-H, Huo X-B, Zhang S-S, Gong X-S. Analysis and study on the population bionomics of the Aedes albopictus in Shandong. Chin J Hyg Insecticides Equip. 2006;12:216-8 (in Chinese).

52. Erguler K, Smith-Unna SE, Waldock J, Proestos Y, Christophides GK, Lelieveld J, et al. Large-scale modelling of the environmentally-driven population dynamics of temperate Aedes albopictus (Skuse). PLoS One. 2016;11: e0149282.

53. Johnson TL, Haque U, Monaghan AJ, Eisen L, Hahn MB, Hayden MH, et al. Modeling the environmental suitability for Aedes (Stegomyia) aegypti and Aedes (Stegomyia) albopictus (Diptera: Culicidae) in the contiguous United States. J Med Entomol. 2017:54:1605-14.

54. Ho SH, Speldewinde P, Cook A. Predicting arboviral disease emergence using Bayesian networks: a case study of dengue virus in Western Australia. Epidemiol Infect. 2017;145:54-66.

55. Eisen L, Monaghan AJ, Lozano-Fuentes S, Steinhoff DF, Hayden MH, Bieringer PE. The impact of temperature on the bionomics of Aedes (Stegomyia) aegypti, with special reference to the cool geographic range margins. J Med Entomol. 2014;51:496-516.
Ready to submit your research? Choose BMC and benefit from:

- fast, convenient online submission

- thorough peer review by experienced researchers in your field

- rapid publication on acceptance

- support for research data, including large and complex data types

- gold Open Access which fosters wider collaboration and increased citations

- maximum visibility for your research: over $100 \mathrm{M}$ website views per year

At $\mathrm{BMC}$, research is always in progress.

Learn more biomedcentral.com/submissions 